\title{
Jejunal Stenosis, CTCAE
}

National Cancer Institute

\section{Source}

National Cancer Institute. Jejunal Stenosis, CT CAE. NCI Thesaurus. Code C143623.

A disorder characterized by a narrowing of the lumen of the jejunum. 